\title{
Fault Diagnosis Method for Heterogeneous Information Fusion of Permanent Magnet Generator Considering Classifier Performance and Weight of Evidence
}

\author{
Xun YIN \\ School of Electrical Engineering, Xinjiang University, \\ Urumqi 830047, China
}

\author{
Shao-ran WANG* \\ School of Electrical Engineering, Xinjiang University, \\ Urumqi 830047, China \\ e-mail: wangshaoran@vip.qq.com
}

\section{Zhi-wen LUO}

School of Electrical Engineering, Xinjiang University, Urumqi 830047, China

\begin{abstract}
Aiming at the problem that the potential fault of the permanent magnet synchronous generator is difficult to be accurately identified, a potential fault diagnosis model based on probability output of multi-class support vector machine (SVM) and improved D-S evidence theory is proposed. Furthermore, the generator stator current and vibration characteristics are applied in the establishment of diagnostic model respectively and the failure probability based on the heterogeneous feature is obtained. Considering the difference of the fault characterization ability between the current evidence and the vibration evidence, as well as the generalization ability of SVM, the weight fusion model is established, and the output of the model is the final diagnosis criterion.
\end{abstract}

Keywords-Permanent magnet synchronous generator; Support vector machine; Mechanical and electrical integrated information; Weight fusion; Potential fault diagnosis

\section{INTRODUCTION}

Wind turbines work in extreme condition, mechanical or electrical failures are always occurred. Recently, wind turbines condition monitoring and fault diagnosis technology are paid more attention. Wind turbine fault diagnosis information comes mainly from single information source. Although some achievements have been made in wind turbine fault diagnosis field, many problems still exist.

In order to make full use of the complementarity of vibration and current information, a potential fault diagnosis method of permanent magnet synchronous generator based on mechatronic information fusion is proposed in this paper. The experimental results show that the diagnosis accuracy is improved compared with the method based on single information source.

\author{
Xin-yan ZHANG \\ School of Electrical Engineering, Xinjiang University, \\ Urumqi 830047, China \\ Lu-lu YANG \\ School of Electrical Engineering, Xinjiang University, \\ Urumqi 830047, China
}

Li-wei ZHAO

School of Electrical Engineering, Xinjiang University, Urumqi 830047, China

\section{MULTICLASS SVM MODEL BASED ON PROBABILISTIC OUTPUT}

\section{A. Probability Output of Binary Class SVM}

Support vector machine (SVM) has a high accuracy and good generalization performance for small sample classification, and it is widely used in the field of fault diagnosis. The output of the traditional SVM is certain fault type, and other fault information is not fully utilized. In this paper, the relationship between SVM output and posterior probability is established by using sigmoid function to realize the posterior probability output of SVM. More details about this method are described in reference [1].

\section{B. Probability Output of Multi-class SVM}

The probabilistic estimation method in section 3.1 can only be applied in binary classification problem. In this paper, we use the method of pair-wise coupling and "one-to-one" multi-classification strategy to extend the probability output of binary classification to multi-classify and realize multiclassification probability output. More details about this method are described in reference [2].

\section{EVIDENCE FUSION METHOD CONSIDERING INFORMATION WEIGHT AND SVM PERFORMANCE}

In this paper, an improved D-S evidence fusion method is proposed. The method takes the sensitivity of evidence as well as the generalization ability of SVM into consideration, and diagnostic accuracy was improved significantly.

The generalization ability of SVM can be evaluated by random cross validation. Define the SVM performance discount factor $\eta$ to evaluate SVM generalization ability. SVM performance discount factor $\eta$ equals to optimal random cross validation classification accuracy.

Suppose identification framework $\mathrm{D}=\left\{A_{1}, A_{2}, \ldots A_{n}\right\}$ contained $\mathrm{n}$ types of possible fault, and every type of fault 
contains $N_{i}(i=1,2, \ldots \mathrm{n})$ fault samples respectively. The samples are input into multi-class SVM and evidence reliability matrix can be obtained:

$$
R E=\left[\begin{array}{cccc}
c m_{11} & c m_{12} & \cdots & c m_{1 n} \\
c m_{21} & c m_{22} & \cdots & c m_{2 n} \\
\vdots & \vdots & \ddots & \vdots \\
c m_{n 1} & c m_{n 1} & \cdots & c m_{n n}
\end{array}\right]
$$

where element $c m_{i j}(i, j=1 \ldots n)$ is the number of samples belonging to class $i$ fault while determined to class $j$ fault by SVM; $\mathrm{Cm}_{i i}$ is the number of the fault samples which are classified correctly. The reliability matrix reflects the relationship between the real fault types and the diagnostic results of each sample and can evaluate the performance of the sub models.

The reliability coefficient of evidence for class $i$ can be defined as the percentage of the number of samples which is diagnosed correctly as fault class $i$ in the number of fault class $i$ which SVM determines. The reliability coefficient of evidence for class $i$ can be calculated as Eq. (2). The evidence weight assignment function is shown in Eq. (3):

$$
\begin{gathered}
R_{i}=\frac{c m_{i i}}{\sum_{i=1}^{N} c m_{i j}} \quad(i=1,2, \cdots n) \\
w\left(A_{i}\right)=\frac{1}{1+e^{-8\left(R_{i}-0.5\right)}} \quad(i=1,2, \cdots n)
\end{gathered}
$$

The relationship between reliability coefficient and weight of evidence is shown in figure1.The weight of evidence on the interval $[0,1]$ is increasing. The greater reliability coefficient evidence gets, that is, the higher reliability diagnose result has; correspondingly, the evidence can obtain greater weight. BPA of each piece of evidence is weighted according to formula (4):

$$
\left\{\begin{array}{l}
W m\left(A_{i}\right)=\frac{w\left(A_{i}\right) \bullet m\left(A_{i}\right) \bullet \eta}{m(D)+\sum_{A_{i} \subseteq D} w\left(A_{i}\right) \bullet m\left(A_{i}\right)} \quad\left(\forall A_{i} \subseteq D\right) \\
W m(D)=1-\sum_{i=1}^{n} W m\left(A_{i}\right) \quad\left(\forall A_{i} \subseteq D\right)
\end{array}\right.
$$

Where $\eta$ is the SVM performance discount factor; $W m\left(A_{i}\right)$ is the BPA of the event $A_{i}$ after weighted fusion; $W m(D)$ indicates uncertainty of faults after weighted fusion, and the results are used as the final diagnostic criteria.

\section{FAULT DIAGNOSIS METHOD BASED ON MECHANICAL AND ELECTRICAL INFORMATION FUSION}

Normal operating conditions $\left(A_{1}\right)$, mild corrosion of bearings $\left(A_{2}\right)$, rotor imbalance $\left(A_{3}\right)$, and stator unbalance $\left(A_{4}\right)$ of permanent magnet generators are discussed in this paper. All samples are divided into training samples, detection samples and testing samples. The diagnosis process mainly contains three steps. The diagnostic process is shown in figure 2 .

\section{A. Step 1: Fault Feature Extraction}

(i) Db5 wavelet basis is used to de-noise and reconstruct radial vibration signal. Standard deviation $(s t)$, kurtosis index $(k u)$, waveform index $(S)$, peak value $(C)$, pulse index $(I)$, and margin index $(L)$ are extracted from the signal as the characteristic parameter. Characteristic parameters are defined in reference [3]; for the high frequency wavelet coefficients, energy of wavelet coefficients $e_{i}(i=1,2, \ldots 5)$ and Shannon entropy $E$ of each layer are calculated respectively. The characteristic parameters mentioned above constitute the 12-dimensional vibration feature vector $F_{l}$ :

$$
F_{1}=\left[s t, k u, S, C, I, L, e_{1}, e_{2}, e_{3}, e_{4}, e_{5}, E\right]
$$

(ii) For the stator current, modulus square current amplitude at 1 times rotor frequency $\left(z_{1}\right)$, DC component in A phase current (relative to the threephase system) $\left(z_{2}\right)$, current amplitude at 2 times fundamental frequency $\left(z_{3}\right)$, current amplitude at 3 times fundamental frequency $\left(z_{4}\right)$, current amplitude at5 times fundamental frequency $\left(z_{5}\right)$, current amplitude at7 times fundamental frequency $\left(z_{6}\right)$ (relative to $50 \mathrm{~Hz}$ fundamental frequency) are extracted to constitute the current feature vector $F_{2}$. Characteristic parameters are defined in reference [4];

\section{B. Step 2: Calculation about SVM Performance Discount Factor And Weight of Evidence:}

SVM fault diagnosis model based on vibration features and current features is established by training samples and 10-fold random cross-validation is subjected to training samples. The optimal classification accuracy rate is used as the SVM performance discount factor ${ }^{\eta}$. Detection samples are put into training model, and evidence reliability coefficients of four kinds of faults $w\left(A_{1}\right), w\left(A_{2}\right), w\left(A_{3}\right), w\left(A_{4}\right)$, are obtained according to formula (1) - (3).

\section{Step 3: Weighted Fusion for BPA}

According to the method mentioned in Section 3, the BPA of evidence is weighted and fused, and final diagnosis result is judged according to the following rules:

(i) Final diagnosis result must have the greatest value of $\mathrm{BPA}$;

(ii) The BPA difference between final diagnosis result and other faults should be greater than a certain threshold. In this paper, the threshold is set to 0.15 ;

(iii) The uncertainty of the diagnostic results should be less than a certain threshold which is set to 0.1 ; 


\section{EXPERIMENTAL ANALYSIS}

The data comes from the permanent magnet generator testing platform in our school. 12 dimensional characteristic parameters of vibration signal and 6 dimensional characteristic parameters of stator current signal are extracted.

The features of training samples are input into fault diagnosis models which are established based on vibration and current signal respectively. PSO algorithm is used to optimize the parameters. The optimal parameter $\mathrm{C}$ about vibration diagnosis model is equal to 945.11 ; the optimal parameter $\mathrm{g}$ is equal to 0.125 ; the performance discount factor $\eta$ is equal to 0.9 . The optimal parameter $\mathrm{C}$ about current diagnosis model is equal to 541.41; the optimal parameter $\mathrm{g}$ is equal to 97.33 and the performance discount factor $\eta$ is equal to 0.867 .

20 groups of four states of vibration test samples are input into the trained vibration diagnosis model, and the reliability matrix $R E_{l}$ based on the vibration characteristics is obtained; 20 groups of current test samples with four different states are input into the trained current diagnostic model, and the reliability matrix $R E_{2}$ based on the current characteristic is obtained:

$$
R E_{1}=\left[\begin{array}{cccc}
19 & 0 & 0 & 1 \\
1 & 18 & 1 & 0 \\
0 & 3 & 17 & 0 \\
2 & 0 & 0 & 18
\end{array}\right] R E_{2}=\left[\begin{array}{cccc}
17 & 2 & 1 & 0 \\
2 & 16 & 2 & 0 \\
3 & 2 & 15 & 0 \\
4 & 2 & 1 & 13
\end{array}\right]
$$

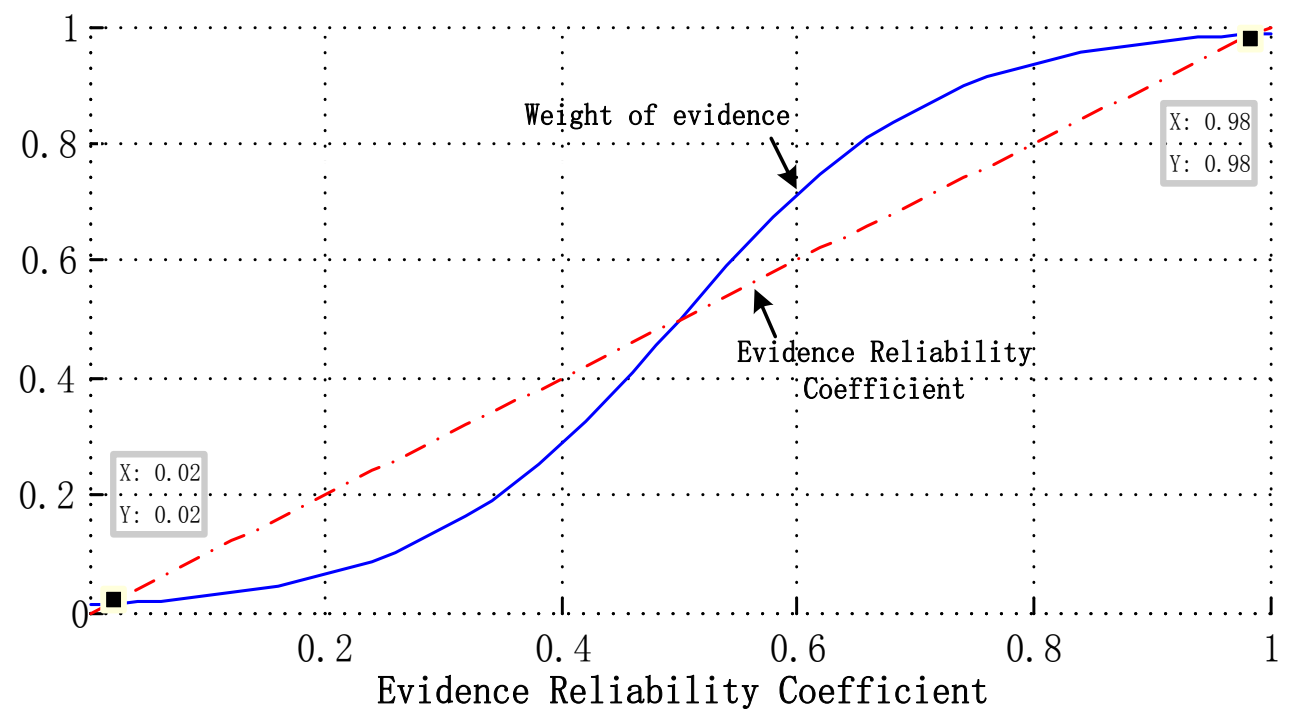

Figure 1. Relationship between Reliability Coefficients

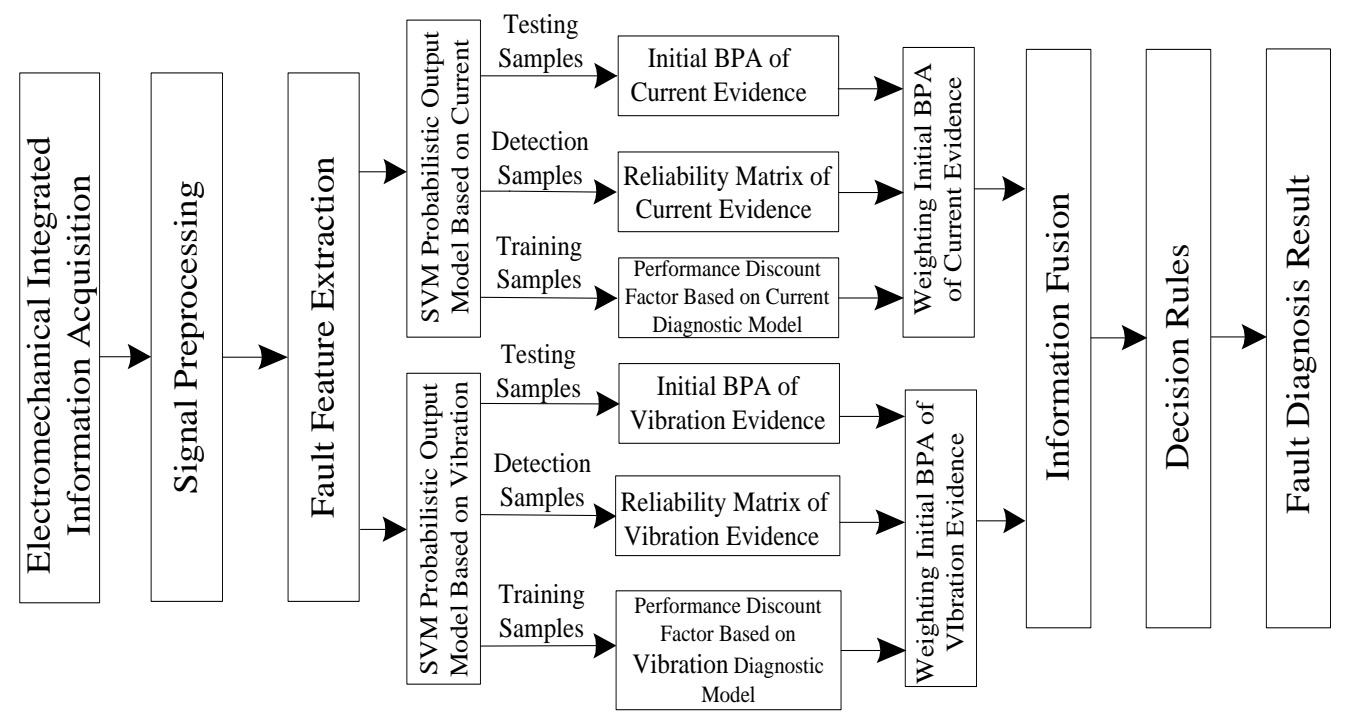

Figure 2. Fault Diagnostic Process 
TABLE I. WEIGHT COEFFICIENTS OF EVIDENCE

\begin{tabular}{ccccc}
\hline Evidence & $w\left(A_{1}\right)$ & $w\left(A_{2}\right)$ & $w\left(A_{3}\right)$ & $w\left(A_{4}\right)$ \\
\hdashline Vibration & 0.948 & 0.946 & 0.972 & 0.973 \\
Current & 0.774 & 0.860 & 0.910 & 0.982 \\
\hline
\end{tabular}

TABle I. COMParison of Diagnostic Results for All Test SAMPLES

\begin{tabular}{|c|c|c|c|c|c|c|}
\hline \multirow{2}{*}{$\begin{array}{l}\text { Information } \\
\text { Source }\end{array}$} & \multicolumn{4}{|c|}{$\begin{array}{c}\text { Fault Recognition } \\
\text { Accuracy }\end{array}$} & \multirow{2}{*}{$\begin{array}{l}\text { Correct } \\
\text { Rate }\end{array}$} & \multirow{2}{*}{$\begin{array}{c}\text { Uncertaint } \\
\mathrm{y}\end{array}$} \\
\hline & 1 & 2 & 3 & 4 & & \\
\hline Vibration & 0.9 & $\begin{array}{l}0 . \\
6\end{array}$ & $\begin{array}{l}0 . \\
6\end{array}$ & $\begin{array}{l}0 . \\
7\end{array}$ & 0.7 & 0.225 \\
\hline Current & 0.8 & $\begin{array}{l}0 . \\
5\end{array}$ & $\begin{array}{l}0 . \\
4\end{array}$ & 1 & 0.675 & 0.075 \\
\hline $\begin{array}{l}\text { Integrated } \\
\text { Information }\end{array}$ & 1 & $\begin{array}{l}0 . \\
5\end{array}$ & $\begin{array}{l}0 . \\
7\end{array}$ & 1 & 0.8 & 0.125 \\
\hline
\end{tabular}

The weight evidence of the reliability matrices is calculated according to formula (12) and (13), and the results are summarized in table 1.40 test samples is input into the trained SVM diagnostic model to obtain initial BPA. After that, the weighted fusion is made according to formula (10) and formula (14).

The diagnostic results of the 40 samples of the whole test set are summarized in table 2. Fault types 1, 2, 3, 4 respectively represent the conditions of normal operation, mild corrosion of bearing, rotor imbalance and stator imbalance. After weighted fusion, the recognition accuracy of each fault is improved, and the uncertainty is maintained at such low level, which proves the validity and correctness of the method.

\section{CONCLUSIONS}

The multi classification probability information is introduced into the diagnosis process and overcome the defects of the traditional SVM diagnosis model. Evidence weighted fusion can make full use of redundant information about vibration as well as stator current and precision of diagnosis are improved.

\section{ACKNOWLEDGMENT}

This work is supported by National Science Foundation of China (51367015)

\section{REFERENCES}

[1] PLATT J C. probabilistic outputs for support vector machines and comparison to regularized likelihood methods [M]. Cambridge, MA, USA: MIT Press, 2000:61-74

[2] HASTIE T T R. Classification by pairwise coupling [J]. The Annals of statistics, 1998, 26(1):451-471.

[3] An XueLi, Zhao Minghao, Jiang Dongxiang. Direct-drive wind turbine fault diagnosis based on support vector machine (SVM) and multiple source information [J]. Power System Technology. 2011, 35 (4): $117-122$

[4] Hang Jun, Zhang Jianzhong, Cheng Ming, et al. Permanent magnet synchronous wind turbine fault diagnosis of impeller imbalance and winding asymmetric [J]. Proceedings of the CSEE, 2014, 34 (9): 1384-1391 